\title{
Composite model of a hematopoietic system with clone competition
}

\author{
M. Rusinov ${ }^{1,2}$, S. Kulikov ${ }^{2}$ \& L. Uvarova ${ }^{1}$ \\ ${ }^{1}$ Moscow State Technological University "Stankin”, Moscow, Russia \\ ${ }^{2}$ National Centre for Hematology, Moscow, Russia
}

\begin{abstract}
This paper is dedicated to the development of a human hematopoietic system model, its identification and investigation of the roles of polyclonal and complex feedback in defining the behaviour of the system. The main purpose of the model framing is the study of the interaction and the possible pathogenetic role of simultaneously coexisting distinct lines of blood, which develop in parallel. The article proposes a classification system for human hematogenesis models based on following a key principle: the method of describing the age structure of cell population. Based on the analysis of said classification certain system features, which have not been considered previously, stand out. To take them into account, we propose a model based on the McKendrick-von Foerster equation. This paper describes the features and limitations of this model and discusses further development of the model
\end{abstract}

Keywords: mathematical model, hematopoietic system, stem cell, hematopoiesis.

\section{Introduction}

Due to the nature of their functioning, blood cells are subjected to constant physiological destruction, which makes the hematopoietic system one of the most dynamic systems. Constant change of the internal and external conditions requires large variability of the system and high efficiency of directional change in the production of blood cells.

Due to the complexity of the regulation of the hematopoiesis even small changes in the behaviour of individual cells can switch the system to other operational modes. 
The fact that many blood diseases are rare suggests that subtle mechanisms are required in order to understand their causes. Heuristic arguments without applying mathematical apparatus of the system study might fail to provide the explanation of the pathogenesis and therapeutic ways of returning the system to a normal functioning condition.

The two main principles determining the architecture of the hematopoietic system are the presence of a regulatory feedback and the change in the cell behaviour depending on their degree of differentiation. If we analyze the currently existing mathematical models of hematopoiesis based on the method of describing the age structure of the cell population (MDASCP), we can classify them as follows:

1. Models with discrete MDASCP: major classes of cells are selected from the population structure, and for each class a law of change and transition to a related class is stated [1, 2].

2. Models with continuous MDASCP: population structure is seen as a continual sequence of maturing cells. This approach provides two principles for further study:

a. Models with discretized MDASCP: the sequence is divided into any number of age compartments, usually into several dozens, and consistent equations are, fairly accurately describing the structure of transitions from one stage of cell development to another, are developed for them. In this case, the system may include several macro-classes, in which the transitions between the classes are organized differently [3].

b. Models with continuous MDASCP: age structure of the population is described by a certain continuous equation [4-8].

\section{The model}

The analysis of present-day knowledge about the hematopoietic system physiology shows that models described above do not take into account the following features of the human hemopoietic system:

1. The presence of two main opposing principles of production adjustment:

a. By regulating the rate of proliferation;

b. By regulating the rate of differentiation;

2. Polyclonality;

3. The behaviour of the hematopoietic system as a cell population.

To construct the dynamics of change in the cell population it appears reasonable to apply an approach of modeling with continuous description of the structure of age distribution in the population. Such a model would consist of integrodifferential equations and can be studied analytically.

We divide the population of blood cells into two blocks fundamentally distinguishable from one another by the nature of behaviour and by a non-trivial 
interaction between them: one block for maturing cells in the bone marrow (BM) and the other for mature cells in the peripheral blood (PB). The block with mature cells influences the dynamics of the maturation of the cells from the block of maturing cells. Transition of the cells from one block to another is random. In addition, the block of maturing cells is also divided into two blocks: block of maturing cells, which under normal circumstances do not go into the peripheral blood, and block of mature cells in BM, which gradually diffuse through the bone tissue into the bloodstream. The transition from the block of maturing cells to a block of mature cells in the BM occurs after a certain degree of differentiation. The transition from the block of mature cells in the BM to the block of mature cells in the PB is uniform. The probability of death of mature cells increases with their age. The total number of mature cells influences the regulatory factor $(\mathrm{RF})$ levels of the feedback.

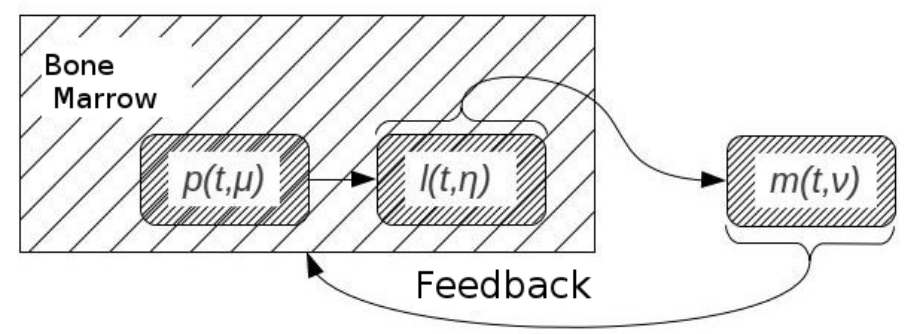

Figure 1: Schema of the model with a continuous description of the age structure of the cell population.

The most plausible description of the population structure of each block can be obtained by using McKendrick-von Foerster equation. This equation is a partial differential equation with two variables: time and differentiation degree of the cells (their relative age), which describes changes in population structure. The left side of the equation contains terms that describe changes in population structure, and the right side contains terms that describe population growth and decrease. For each block a relative cell age is introduced and the global time is implemented.

For each block we must determine the right side of the equation, depending on the laws of change of the population structure:

1. For the block of dividing and developing cells:

$$
\frac{\partial p}{\partial t}+V(D) \frac{\partial p}{\partial \mu}=\beta(\mu, P) p=s_{0} P \exp \left(-\frac{\mu}{k_{\beta}}\right) p
$$

where $p(t, \mu)$ is the maturing cells population density; $V(D)$ is the factor responsible for the rate of cell differentiation, which depends on the level of the differentiation regulation factor (DRF) $D(t) ; \beta(\mu, p)$ is the coefficient of cell proliferation rate, which depends on their maturity and the level of the proliferation regulation factor $(\mathrm{PRF}) \mathrm{P}(\mathrm{t})$. 
As the differentiation continues, the cells lose their proliferative potential. It is marked by the acquisition of specific morphological traits.

For this relation the following function is suggested:

$$
\beta(\mu, P)=s_{0} P \exp \left(-\frac{\mu}{k_{\beta}}\right)
$$

where $s_{0}$ is the proliferative potential of the cell that has entered the path of the development (right after the "stem"); $P$ is the level of PRF; $k_{\beta}$ is a scale factor.

The rate of the cell division depends on the concentration of DRF D(t) and on its enzyme kinetics, which are implemented according to the Michaelis - Manton equation:

$$
V(D)=\frac{V^{\max } D}{D+K_{m}}
$$

where $\mathrm{V}^{\max }$ is the top speed of maturation; $\mathrm{K}_{\mathrm{m}}$ is the Michaelis constant, correspondent to the enzyme concentration at which the rate of the cell maturation equals half of the maximum. $\mathrm{S}_{0}$.

The initial condition for the eq. (1) is a constant number of stem cells $\mathrm{p}(\mathrm{t}, 0)=$

At maturity level $\bar{\mu}$ cells lose their ability to divide, but continue to mature in the bone marrow and other hematopoietic organs. In our model, we represent it by gluing the boundary conditions of the 1st block (right) and the 2nd (left).

$$
l(t, 0)=p(t, \bar{\mu})
$$

2. For the block of mature cells in bone marrow:

$$
\frac{\partial l}{\partial t}+V(D) \frac{\partial l}{\partial \eta}=V(D) H(\eta) l=-\frac{V(D)}{\lambda_{H}} l
$$

where $l(t, \mu)$ is the mature cells population density; $V(D)$ is the factor responsible for the rate of cell differentiation, which depends on DRF $D(t) ; H(\mu)$ is the function of the cell transition rate in peripheral blood.

In the time of transition from the first block to the second the cells acquire the potential to pass into the peripheral blood. Such processes are described by the Weibull distribution: the conditional probability of a cell passing into the peripheral blood, provided that by current age it has not gone there yet, that is, the risk function. The probability of passing for each cell is independent of its age, $\mathrm{kH}=1$, hence,

$$
H(\eta)=\frac{k_{H}}{\lambda_{H}}\left(\frac{\eta}{\lambda_{H}}\right)^{k_{H}-1}=\frac{1}{\lambda_{H}}
$$

The function $l(t, \eta)$ is defined on a semi-infinite axis $0 \leq \eta<\infty$ 
3. For the block of mature cells in peripheral blood:

$$
\frac{\partial m}{\partial t}+W \frac{\partial m}{\partial v}=-\gamma(v)=-\frac{k_{\gamma}}{\lambda_{\gamma}}\left(\frac{v}{\lambda_{\gamma}}\right)^{k_{\gamma}-1}
$$

where $\mathrm{m}(\mathrm{t}, \mathrm{v})$ is the population density of the mature cells in the peripheral blood, which are gradually removed from the circulation; W is the rate of "aging" of the cells; $\gamma$ is the rate of cell death.

We assume that the probability of cell death increases with age linearly due to the impossibility of regeneration. Then $\gamma$ is described by the Weibull distribution with $\mathrm{k} \gamma>1$.

The size of the cell inflow to the third block should equal the flow of cells leaving the second block; it equals the age integral of the product of risk function by population structure function.

$$
m(v=0)=\int_{0}^{\infty}(l(t, \eta) H(\eta)) d \eta=\frac{1}{\lambda_{H}} \int_{0}^{\infty} l(t, \eta) d \eta
$$

The system of quantity regulation maintains a constant number of mature cells.

$$
M(t)=\int_{0}^{v_{F}} m(t, v) d v
$$

The total number of mature cells is calculated by their task performance, that is, by their efficiency.

Since RFs are proteins, they are produced and decomposed with time according to the first order kinetics, and if their growth is dependent on the number of mature cells, their decay rate is constant.

$$
\frac{d E}{d t}=f_{E}(M)-K_{E}^{12} E
$$

where $\mathrm{E}$ is one of the RFs, $\mathrm{P}$ or $\mathrm{D}, f_{E}(M)$ is the $\mathrm{E}$ production rate, $K_{E}^{12}$ is the half-life period.

The production of RFs is carried out by special cells, and according to the principles of feedback RFs are produced during cell shortages and stop being produced in the conditions of overabundance.

$$
f_{E}(M)=\frac{E^{\max }}{\left(\frac{M}{q}\right)^{m}+k_{f}^{E}}
$$

where $M(t)$ is the number of mature cells; $E^{\max }$ is the maximum rate of increase of the RF percentage; $m_{E}$ is the Hill coefficient, in this case it reflects the 
severity of the reaction to the deviation from the norm; $\mathrm{q}$ is a scale factor, a shift in the normal level to the inflection point of the kinetic function; $\mathrm{k}_{\mathrm{f}}^{\mathrm{E}}$ is the smoothing coefficient.

Present-day ideas about the physiology of hematopoiesis postulate the existence of a polyclonal hematopoiesis, thus stating the heterogeneity of the population, the presence of a subpopulation with dynamic parameters differing from the main one. Also, the descendants of different cells, identical in their characteristics, can be attributed to the main population.

In general, any of the dynamic parameters of the cell can be changed. If we assume that there is only one changed subpopulation competing with the main, it is advisable to monitor the fate of the cells of both populations. For both hematopoietic lines the equations remain identical up to the values of the coefficients. RF kinetics of the feedback in a polyclonal model does not change in comparison with the model of a homogeneous population. Changes apply only to the formula for $\mathrm{M}$, which calculates the total number of mature cells in the peripheral blood.

The total number of mature cells is calculated by their task performance, that is, by their efficiency, which may be different for different lines, so we introduce the coefficient of efficiency ratio $\Omega$.

$$
M(t)=\int_{0}^{v_{F}} m_{1}(t, v) d v+\Omega \int_{0}^{v_{F}} m_{2}(t, v) d v=\int_{0}^{v_{F}}\left(m_{1}(t, v)+\Omega m_{2}(t, v)\right) d v
$$

However, there is a certain shared resource (food, space etc.) that restricts their joint growth and provides mutual oppression.

\section{The stationary solution}

The resulting model is analytically solved only if $\mathrm{V}(\mathrm{t})=$ const. This case corresponds to a situation where the feedback is not activated, i.e. it is the case of a normal system functioning. The equations of the model are then divided into several independent quasi-linear first order partial differential equations, each of which can be solved by the method of characteristics. As a result, we obtain the structure of the cell population in a situation of normal hematopoiesis. These equations can be used to set the initial conditions for the numerical calculation.

$$
m=S_{0} \exp \left(1-s_{0} k_{\beta} \frac{P_{C}}{V_{C}}\left(e^{-\frac{\bar{\mu}}{k_{\beta}}}\right)-\frac{1}{W}\left(\frac{v}{\lambda_{\gamma}}\right)^{k_{\gamma}}\right)
$$

In addition, on the basis of some a priori reasons several settings that are difficult to formalize can be excluded.

First we need to understand the kinetics of the feedback factors eq. 11. Feedback functioning should lead to the regulation of the blood cell production in a way that would maintain a constant number of mature functioning cells in the blood. Thus, the macro-argument which specifies the whole behavior of the 
hematopoietic system, but is not actually presented in the model, is the normal number of cells in the peripheral blood $\bar{M}$.

The kinetics function of the feedback factors must have such properties that if the value of the cell number in the peripheral blood is normal, the factor will maintain the average value, while a deviation from the normal value will cause a drastic change in the factor production, although a strong deviation will eventually cause saturation, i.e. an asymptotic approach of the production rate to the maximum possible value on one side and to zero on the other.

All this is achieved by the accordance of the $\bar{M}$ value with the function inflection point eq. (11) and the concordance of $\mathrm{E}_{\max }$ with $\mathrm{K}^{\mathrm{E}}{ }_{1 / 2}$

Unlike many other parameters, $\mathrm{K}_{1 / 2}^{\mathrm{E}}$, which is the half-life period of the factor, can be found directly. A maximum rate of the factor production must be obtained indirectly.

To obtain normal values of the factors it is necessary to regard the obtained solution of the stationary problem

\section{Numerical study}

Numerical study has confirmed the presence of limit cycles, as well as damped oscillatory solutions corresponding to the state of blood loss.

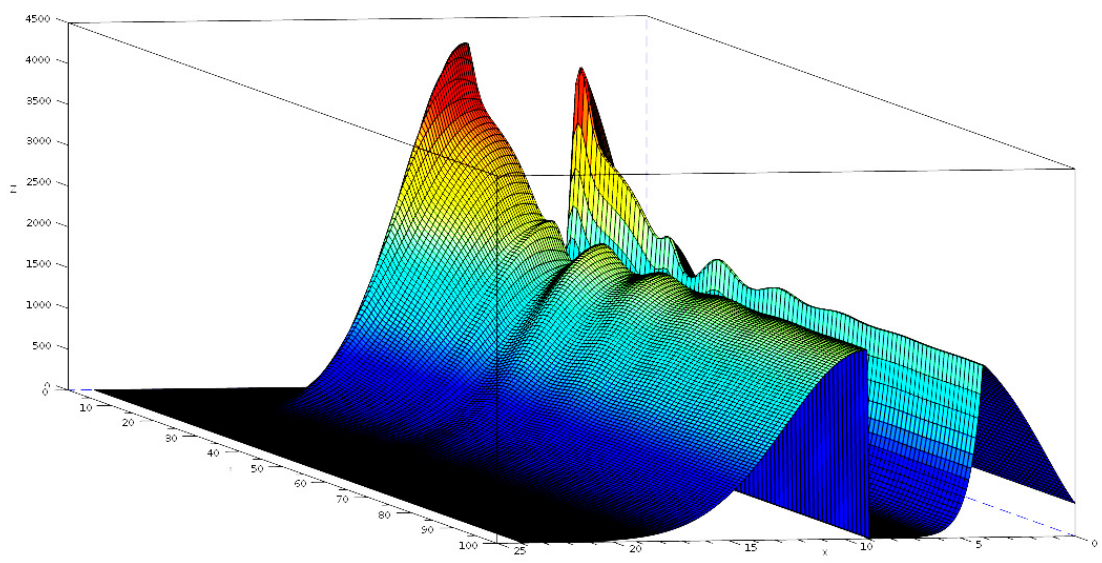

Figure 2: Numerical solution. Blood production is repaired after a massive blood loss. X-axes (reverse) represent the maturity level of cells; Yaxes represent the time; $\mathrm{Z}$-axes represent the number of cells.

\section{Simulation part}

Proposed approach, however, does not allow to fully trace the events occurring, firstly, in the early stages of differentiation, where the behavior can not be described by the "average" behaviour, and, secondly, in "rare" events, such as 
neoplasms of various origins. Rare events in differential models end up outside of the error limits.

When considering additional systems of equations (as suggested above), we face the problem of scale differences in the initial period, as well as, more importantly, the impossibility to investigate the likelihood of development or elimination of the arising mutant clones. In turn, the lack of dynamic equations for further development of the cells does not provide a plausible picture. The feedback in this case makes a significant contribution to the development of the disease.

To model the dynamics of the early stages of hematopoiesis we suggest the use of the Moran model [3, 9]. Moran model is a model with event transition. We believe that the events occurrence is relatively uniform over time, and at no point in time do two simultaneous events occur. It is always possible to find the average time between events. In this case, that period $\tau$ is inversely proportional to the volume of the given population and is directly proportional to the average time of single cell events. We will consider division, apoptosis and differentiation as such events. We will look at the first few compartments. Starting with a certain maturity level $\mathrm{T}$ (determined based on the volume of the compartment) the events can be considered as deterministic and we can calculate them in our models "averagely" using the differential equations. For the changed clone the probability of events is different from the general population, which by assumption is the cause of neoplasia.

A similar approach is used in [3], but in this study the effect of the feedback is not considered, although it turns out to be essential, since it has an enhancing effect. The resulting estimates may be too low.

Time $\tau$ between events defines the model time for further calculations and subsequent compartments.

To obtain a full calculation model it is necessary to add the equation of displacement into the continuous model. The reaching of the saturation limit can be conventionally considered a sigmoidal function.

\section{Conclusions}

Therefore, we have a combined model where the estimates for the early stages of cell development are carried out basing on Moran law, i.e. individually for each cell, which allows us to assess general probability of pathological process appearing and their development into a disease. And vice versa the solution for a reverse population problem, when we can determine the probability of pathological cell emergence knowing the frequency of pathologies in a cell population. At the same time this phenomenon is non-linear. At the top levels of the system an analytical system is used, which investigates the behaviour of the system on average. The methodology of this approach is viable as it the feedback regulation factors are evenly distributed among hematopoetic organs, and the events involving stem cells are discrete. We view such description of the system as the most credible and complete. 
Analytical solutions available allow us to divide the areas, where trajectories of numerical solutions come, into classes of solutions. This provides an opportunity to select the correct strategy of numerical analysis, in particular it will allow us not to consider impossible trajectories or those falling into the infeasible regions.

The model may serve as a basis for the analysis using Mote-Carlo method as well as for prediction of responses to various therapeutic interventions (treatment protocols) or exposure to medicine.

The model can be further complemented with detailed mechanisms of enzyme kinetics for feedback regulation factors, while Moran model may be replaced with a special model which will allow us to take the interaction between stem cells and microstroma into account.

\section{References}

[1] Pertzev N., Marchuk G., Mathematical model of hematopoiesis. Preprint Computing Center of the USSR, 255, 1980, In Russian.

[2] Belair J., Mahaffy J.M., Variable maturation velocity and parameter sensitivity in a model of haemotopoiesis. IMA Journal of Mathematics Applied in Medicine and Biology, 18, pp. 193-211, 2001.

[3] Lenaerts T., Pacheco J. M., Traulsen A., Dingli D., Tyrosine kinase inhibitor therapy can cure chronic myeloid leukemia without hitting leukemic stem cells. Haematologica, 95(6), pp. 900-907, 2010.

[4] Lyapunov A., Pavlov A., On the construction of mathematical models of eritropoesis. Some problems of mathematical biology: Novosibirsk, USSR, pp. 31-42, 1973, In Russian.

[5] Belair J., Mackey M., Mahaffy J. M., Age-structured and two delay models for erythropoiesis. Mathematical Biosciences, 128, pp. 317-346, 1995.

[6] Mahaffy J. M., Belair J., Mackey M. C., Hematopoetic model with moving boundary condition and state dependent delay: Application in erytropoiesys. J. Theor. Biol., 190, pp. 135-146, 1998.

[7] Mahaffy J. M. Age-structured modeling of hematopoiesis, technical report, Centre recherches mathematiques: Universite de Montreal, CRM-2609, 1999.

[8] Colijn C., Mackey M.C. A mathematical model of hematopoiesis. J. Theoret. Biol., 237, pp. 117-132.

[9] Moran P. A., The Statistical Processes of Evolutionary Theory. Oxford, Clarendon Press, 1962. 